than the vessels themselves, the diameters of wlich are on an average not more than $\frac{1}{300}$ th of an inch.

From this description of the minute anatomy of the lungs we conclude that these organs are nothing more than the vast expansion of a fine, delicate membrane, upon the opposite sides of which blood and atmosphere are sitnated; and that the entire mechanical arrangements we have followed out are simply intended to insure those agents being brought together in such a manner, that by their mutual chemical reaction the black blood of the pulmonary artery may be converted into the bright-red blood of the pulmonary veins. This is the sum and substance of the process of respiration; and whenever any impediment exists which more or less excludes the air from the lung.cells, or any cause is in operation to produce an inordinate accumulation of blood in the capillary plexuses of the pulmonary artery, Nature attempts, by increased inspiratory efforts, to balance the disproportion between the air and the blood.' Should the attempt be attended with some difficulty, a peculiarly distressing sensation results, (dyspncea;) or if the balance can be in no way restored, death from suffocation is the consequence, (apnœa.)

This is a very brief summary of the doctrine of dyspnoa; but I shall have again to revert to it in the course of these lectures.

\section{A Clímícal $\mathbf{z}$ ecture}

$$
\text { ox }
$$

\section{EPILEPSY FOLLOWING INJURY TO THE CRANIUM. \\ Delivered at St. Thomas's Hospital.}

BY SAMUEL SOLLY, Esq., F.R.S.

Wrulrax $\mathrm{O}-$ aged fourteen, a healthy-looking and intelligent boy, was admitted into George's ward on Dec. 1, 1849, under Mr. Solly. His health has been generally very good. Had measles and scarlatina some years ago, but both very slightly, and for three or four years before the accident occurred he was in perfect health. His family are all healthy, and are none of them subject to fits.

In the early part of November, 1848, whilst pulling at a rope against others, his opponents suddenly let go their hold, and he fell backward, striking his head with great violence on the even surface of the flagstones. He was stunned by the blow, and remained insensible for about a quarter of an hour: during this time no convulsions occurred, and in a very little while longer he had quite recovered. There was no wound of the scalp. The accident occurred about five o'clock in the afternoon.

On the morning of the third day afterwards, he suffered from headach and drowsiness, accompanied with thirst and heat of skin. About two p.M. on the same day he was scized with an epileptic fit. The following is an account of it, derived from Mr. Bury, the surgeon who attended him:--"The cause of this fit seemed at the time very obscure, the circumstance of the fall having been kept back from me; there was no indigested substance in the stomach or bowels to account for it, neither was there evidence of disease working within the cranium; the configuration of the head was proper and healthy the fit was very severe, and four or five hours elapsed before the boy was conscions. Leeches were applied, and an evaporating lotion, with the clipping of the hair and pretty active purgative medicines, low diet, cool room, and exclusion of light, \&c. The case did remarkably well." Additional particulars were obtained from the patient himself. He says, that on the accession of the fit he uttered no cry, and he asserts that it lasted three quarters of an hour. He remained well for three weeks; he then went to Bury St. Edmunds, where, during a six weeks' stay, he had six fits. After his return home he remained free from them for six months, when he had another, and also one at the expiration of other four months; the last happened about a month after this, and about ten days before admission. This exceeded in severity any of the other fits, and was not so soon recovered from. A severe headach always precedes, which he describes as commencing at the upper angle of the occipital bone. During the intervals lie is in good health, but has headach when he reads much.

On admission he was in good health. Appetite good; bowels regular; tongue clean and moist; pulse 80, full and compressible; free from headach. At the superior angle of the occipital bone is a depression, trilateral, and painful on pressure; from it commences the premonitory headach.

Dec. 10th.-Has continued well no to seven this morning, when he complained of headach; referred to the frontal region. At half-past seven A.M., a slight epileptic fit came on, preceded by no cry; there was little struggling, the eyes were rolled about, the teeth closed, but not grinding; the tongue not bitten, and no foaming at the mouth; it lasted fifteen minutes, and was succeeded by incoherence, but not coma. He laughed both during and after the fit. At ten A.M. he was quite rational; he was free from headach; the face was rather more compressible than usual, and the pulse was unaffected.

Gentlemen,-The case you have just heard related is one of great interest: it is a case of epilepsy induced by an injury of the head. The exact amount of that injury it is impossible to decide. Just at the superior angle of the occipital bone there is a deep fissure. This might arise from the depression of that angle of the bone. This portion, though not large enough to produce symptoms of compression, might act as a source of irritation. If we could be certain that such was really the case, there could be no doubt as to the course of treatment which ought to be pursued-namely, to apply the trephine, and remove it. This operation is, however, far too serious to be adopted without greater certainty than we possess at present. It will naturally occur to you, how is it possible that there can be any such serious source of irritation constantly in situ, and the result of that irritation only occurring every three or four weeks, the boy, in the interval, remaining perfectly well? Now, strange as this may appear, we do know of parallel cases. On the other hand, it is quite possible, and, on the whole, more probable, that the result of the blow has been an injury to the dura mater, productive of some thickening and deposit. The tenderness of the spot, and the pain which the boy suffers when it is pressed upon, shows that there is some mischief there still. If, however, the disease is in the dura mater, and there is no displacement of bone, then we have a chance of touching the disease by means of mercury and local treatment. In the hope that this view of the case is correct, I have ordered small doses of mercury, a poultice over the spot, and counter-irritation below it over the occipital bone.

Injuries of the head are sometimes followed immediatelythat is, within a few hours-by convulsions, and when such is. the case, your prognosis must be most unfavourable, for they generally indicate some lesion of the cerebral substance. But if the convulsions do not occur for some days, they generally are dependent on subsequent inflammation. All such cases require active treatment, and they will occasionally recover. The following case is in point, and shows what may be done by antiphlogistic measures.

I have never before seen convulsions of such frequency and severity following injury of the brain without a fatal result. That the cerebral lesion must have been very serious is, $I$ think, proved by the paralysis of the arm and leg. In a therapentic point of view, it is important to observe how obstinate the symptoms were until the system was influenced by the mercury. I have no doubt but that the bloodletting arrested the inflammatory action until the mercury performed its work, but it did not appear as if the bloodletting alone could control the disease.

Case-Thomas Smith, a boy, aged fourteen, residing at Woolwich, was admitted into George's ward, under the care. of Mr. Solly, with a severe injury of the head. It was stated: that he had fallen from a scaffolding twenty feet or upwards in height, pitching upon his head; when picked up was quite insensible, and evidently suffering from a severe concussion of the brain. His head was directed to be shaved, a cold lotion to be applied, and warmth to the extremities, and five grains of calomel were given immediately. At half-past one he was again seen by Mr. Solly, when he bad recovered his senses, but was very drowsy, and comlpained of pain in his head; the pulse was somewhat slow and labouring: the pupils dilated, particularly the left, but contracting upon the admission of light. He was ordered to take two grains of calomel every two hours, and to have twenty leeches applied to the head. At nine P.M. was much the same as in the middle of the day; was bled from the arm to eight ounces.

May 12th.-Half-past eight $\Delta . M_{0}$ : He complained still of 
pain in the head, continued drowsy; pulse 72 ; bowels not opened, although he had taken eight doses of mercury. Ordered calomel, five grains, every hour, until the bowels were reliexed. Leeches to be repeated, but thirty in place of twenty.- One p.M. : Continue treatment.-Eleven P.M. was sleeping; sensible when roused, but complained of pain, particularly on the left side of the head. Bowels still confined; had taken nine five-grain doses of calomel. Ordered an enema to be administered immediately, and repeated if necessary. The calomel to be omitted until the morning.

13 th.- Sleeps a goo I deal, and he is not easily roused, but quite sensible when awakened. Said, "I have got the headach, Sir," and then dozed off again; pulse 64; bowels opened twice by the enema, stool lumpy, and dark brown. Ordered, calomel, five grains.-One p.M.: Rather more drowsy, becoming forgetful; leeches to be repeated, thirty in number.-Seven P.M.: The sister observed that he had slight grating of the teeth, and almost immediatcly thrust his tongue from his mouth, and it remained out; at the snme time the right arm was drann up, and there was rolling of the eves. The sister thought he was conscions, but he conll not speak. She then sent for MIr. Solly, which was at eight p.x. His countenance had become more anxious; he was not so readily roused, and answered less perfectly questions put to him, speaking in a drawling way; pulse 76 , not so full. A vein was opened in the arm, but tho blood, which was very dark, flowed so slowly that the temporal artery was opened. He was raised from the pillow, and as soon as little more than an ounce had flown, he had a convulsive fit; the struggle was slight, and accompanied by a slight moan. The artery was then completely divided, which stopped the bleeding, and he was again laid on the pillow; he almost immediately recovered his conscionsness; his countenance was pale, and covered with cold swcat; pulse 56 , irregular. In a few minutes he seemed better, and said, in answer to a question, that he was easier. Ordered, mercury and challs, two grains every four hours.

14th.-Early morning: Said he was very bad; quite conscious; has had no more convulsions; complained of his head; pulse 72 ; soft; mouth rather tender. - Nine p.r. Countenance more cheerful; head cooler; pulse 80 ; said he was better. Continne treatment.-Eleven p.r.: Had another convulsive fit, which was very short; foaming from the month, which was drawn somewhat to the right side; no scream. A few minutes before this he had started up in the bed, and would have fallen out but for the attendant.

15th.--Several fits, of short duration, have occurred during the night.-Half-past eight : Countenance very heavy and dull; scarcely any answer given to questions; right side of face and arm partially paralyzed, but can feel somewhat when pinched; bowels not open. Cuntinue the mercury and chalk. -Half-past seven P.M.: Has had several fits, but not of great length, nor severe; countenance much the same; answers slowly, but rationally; bowels relieved; motions loose and green; pulse 80 . Continue the mercury and chalk.

16 th. - Not so conscious, or so well in other respects; pulse eighty. Continue treatment.

$17 \mathrm{th}$.- Very drowsy; has frequert fits, some of longer duration than before; countenance heavy and bad; pulse 140 cannot get him to take food; is not aware when it is offered him.

18th.-Much worse; countenance continues anxious; can scarcely answer any question; pulse 156 , small; says he wants his breakfast; always expressing a feeling of hunger. Convulsions occur very frequently.

19th.-Countenance more anxious; says he is better, and that the pain in the head is less; has greater difficulty in speaking, and is certainly worse. Continue mercury.

20th.-A ppears much the same; says he feels bettor; pupils both act to light; the right arm is paralyzed, but not the leg, the convulsions continue.

21 st.-Convulsions not so frequent in occurrence, but continue for a greater length of time, and are more violent; he is not so drowsy; his countenance has improved, and he says he has less pain in the head; pulse 130; bowels open. Continue treatment.

22nd.-The convulsions continne very violent, but occur less frequently even than yesterday; his countenance has mueh improved; pupils act; pulse 120; bowels open; says he is better, but complains of his head.

$23 \mathrm{rd}$.- There is little alteration as regards the frequency and violence of the convulsions; he has the power of moving his arm, which was paralyzed on Wednesday; courtenance continues good; pulse 120, bowels open; mouth beginning to get a little tender. Continue treatment.
24 th.- Has lad no fit since last night; appears much relieved; the pain in the head diminished; countenance good; bowels open; tongue cleaning; pulse 112; complains of his gums. Continue treatment. Apply cold lotion to the head. Hns quite recovered the power of his arm; pulse 108; bowels open; appetite gcod. Continue the pills njght and morning, instead of "very sixth hour. Discontinue the application of the ointmont.

20th.- Iras passcd a good night; no recurrence of the fits; pain in the head slight, and confined to the frontal region; bowels open; pulse 108. Mouth tender.

27 th. - Says he is better; has but little pain in the head; tongue cleaning; pulse 112; bowels confined. Ordered, compound colocynth pill, ten grains, to be repeated if necessary. IIis bowels were relieved before the colocynth was given, therefore no yurgative required.

2sth.--Is progressing fivourably.

June 3rd.-Hlis gums are still tender, and he is generally much better. Ordered, mercury with chalk every other night, instead of night and morning.

Tth.-Is much improved; has no pain in the head, or elsewhere; bowels regular; tongue clean; appetite good; pulse 90.

13th.-Appers and describes himself as feeling quite well; is rapidly gaining strength. To discontinue the pills altogether.

This lad recovered perfcctly.

When the convulsive paroxysms occnr at regular intervals, leaving the patient perfectly well in the intervals, they then constitute the disease which we call epilensy, and on this disease I must say a few words.

Epilepsy is a subject which has engaged the anxious attention of the profession from the first dawn of medicine as a science up to the present time. Accidental circumstances have bronght the disease a good deal before my personal ob. servation, and though we may each of us have our particular theory of its nature, it must still be allowed that we are almost as much in the dark rcgarding its true pathology as they were in the day of Hippocrates. In all cases I believe that the con. vulsion is preceded by a temporary hypergmia, or flush of the cerebral vascular gystem. It is true that this theory has its difficulty. For instance, many cases of epilepsy, especially in females, appear to depend on general anæemia; but it must be remembered that there are also cases of amenorrhca, and that when the utcrine functions are restored, the epileptic paroxysms disappear also. It has been asserted that convulsions in infants are induced by these two totally opposite con. ditions; but this I doubt. I do not mean that I doubt that convulsions in infunts are connected with a general anæmic as well as hrperæmic condition; but $Y$ believe that the vascular system of the brain and spinal cond must be the same in al cases immediately preceding the conrulsion. I cannot believe that similar phenomena can be produced by totally dissimilar causes. But we all know that local congestions and local inflammations occur in totally opposite conditions of the vaseular. system; but the direct effect of the congestion or inflammation is the same. Take, for instance, strumous ophthalmia of the eye in a miserable half-starved child, and active ophthalmia in a plethoric man. The immediate effect on the sight is quite the same in both. I have observed that $i$ 's son e pstient. and these have been young men in the prime of life, with florid complexions, the fit has been immediately preceded by a flush of the facc. In others, and these are chlorotic females suffering from amenorrhcea, the fit is preceded by a deadly pallor. Again: you will often find, in taking the history of the rise and progress of a case of epilepsy, that previous to a true epileptic fit, the patient has sutfered from repeated faintings. This is a very important fact, and one you must attend to, as it may enable you to avert the complete epileptic disease by previous treatment promptly applied. In the first case it is possible there is blood enough in the body to flush both external and internal vessels; both the vessels of the brain and the vessels of the face; in the other, the supply being limited, the brain alone gets an undue quantity.

I believe, then, that the morbid action which precedes an epileptic attack is a sudden deternination of blood to the brain, which expends itself in the secretion of that nervous power, which, in a state of health, is employed by the brain to convey volition to the muscles, which power is, I have no donbt, identical with electricity. This excessive secretion is carried by the motor nerves like a discharge from an electric battery, and from its ruantity and excess produces excessive action of the muscles. It is another illustration of a law which I have had occasion to speak about before-namely, that the 
first effect of arterial excitement in every secreting organ is to excite to an unnatural degree the natural function of the organ. We know that mental emotion will cause a sudden determination of blood to other organs, which, according to the part, will be followed or not by secretion.

Blushing and erection of the penis are instances of sudden determination of blood to a particular part. And the lacrymal glands, salivary glands, testicles, prostate gland, gastric glands, and even the kidneys often pour forth their secretions so abundantly, and so suddenly, that the formative fluid, the blood, must have circulated through their capillaries in greater quantity, and with greater rapidity, than when the glands were at rest, and their secretions suspended. I think that the periodic attacks of mania with which many of the insane are afficted may be regarded in this light.

The vessels which are especially the seat of this morbid action, I suspect, are those of the plexus choroides and one of the laycrs of the cortical substance. The choroid plexus is frequently found hypertrophied in the brain of epileptics, assuming an almost fleshy appearance. This hypertrophy would very probably be the effect of repeated action. It is also the seat of small tumours, generally like hydatids.

The expression "determination of blood to the head" is often made use of, but without any explanation of the manner in which this takes place. I doubt whether the profession generally have any distinct idea as to the exact condition of the vascular system that produces it. I would venture to offer the following theory, the first idea of which I certainly derived, many years ago, from that most truly philosophical work, the "Elements of Physic" of Dr. Arnott. It applies, not merely to the head, but everywhere else.

The middle or muscular coat of the arteries, in a state of health, contracts, with each systole of the ventricles, just sufficiently to give a solidity to the wall of the pipe; so that the force of the contraction is not lost on a yielding surface; a much greater force is required to drive water through a leather hose than through a leaden tube. The middle coat contracts sufficient to assimilate the artery, physically and temporarily, to the leaden tube. Arteries with permanently rigid walls, like leaden tubes, would have interfered, by their rigidity, with the motions of the limbs; and hence this beautiful contrivance. When this middle coat does not contract, or only contracts imperfectly, then the force of the heart dilates the tubes and produces congestion.

I believe, then, that determination of blood to the head arises simply from deficient contraction of the muscular coat of the capillaries of the brain, preceded by the excitement of the heart's action.

The throbbing of the carotid arteries may be considered as corroborative evidence in favour of this opinion. The throbbing cannot arise from action of the vessel; it is the action of the heart, felt strongly, and seen distinctly, because the tube yields to the impulse of the left ventricle, instead of resisting it, like a solid leaden pipe. If the throbbing arose from the action of the artery, it would not be synchronous with the heart, which it is. It is the same yielding of the coat of the capillaries, in an inflamed limb, which gives rise to the throbbing sensation which all of us have felt in some small spot or another.

$I$ think that it is not at all improbable, that the reason why these capillaries of the brain thus suddenly and unnaturally neglect to perform their duty is some defective innervation from the sympathetic nerves, whose office I hold to be the regulation of the coat of the arteries, so as to produce secretions \&c.

There are many circumstances attending organic disease of the brain which I think can only be accounted for on the supposition that the quantity of blood in the brain varies very much at different times. One of the most striking of these is the remission of pain and accession being produced by anything that would tend to accelerate the circulation.

Most authors who have written on epilepsy, Esquirol among the number, agree in stating that the brain is in a state of congestion during the fit.

The amazing benefit which $I$ have seen derived from the use of digitalis is strongly corroborative of this theory. This powerful medicine was most serviceable when it kept the pulse down even below the standard of health.

$I$ believe that in all cases of fatal epilepsy, where there has been autopsy, the vessels of the brain and membranes have been found enormously distended, and in some there has been extravasation.

The warning which many epileptics have-viz, a singing noise in the ears, I believe arises from the dilated carotid artery vibrating in the carotid canal, close to the vestibule of the internal ear.

I regard it as analogous to the throbbing produced by the dilated artery in inflamed parts.

Dr. Conolly observed that epileptic patients are occasionally warned of the approach of paroxysm by mental excitement, their high spirits becoming to their friends the wellknown precursors of their sufferings. This must arise from arterial action. It is analogous to the mental excitement in duced by spirituous liquors; no one doubts that this psychical effect is produced by a physical cause.

Dr. Bright says-" I believe that almost always, during the epileptic paroxysm, either as a cause or an effect, sanguineous congestion takes place within the brain."

Dr. Prichard's and Dr. Alison's opinions coincide with this. But I must not detain you longer on this branch of our subject, as I have entered very freely into it in my work on the brain.

For a knowledge of all the medicines which have been used in the treatment of this formidable disease, I must refer you to Dr. Copland's admirable and erudite article on this subject. In some cases, as, for instance, in the case which has called forth these remarks, there are general synjptoms which call for especial treatment; but there are many cases of epilepsy which are clearly functional, and then your treatment must be, to a certain extent, experimental or empirical.

In females suffering from amenorrhcea, I have seen the greatest benefit derived from the use of steel. But this mineral must be continued for months,-obstinately continued. In order to do this, you must vary the form of it, always giving it in small doses, or you will nauseate your patient, and disturb the digestive organs. The carbonate of iron, the iron mixture, the citrate of iron, the sulphate of iron, are all useful in their turn. I have lately had a suecessful case, by obstinately persevering in this plan of treatment, in the person of a young lady, who had suffered from this complaint for above five years, and who had been under the care of many of the physicians at the West-end. She has now been free from at tacks for nearly two years.

After administering steel for some time, great advantage is often derived from the combination of the sulphate of iron and sulphate of zinc. In young men with a florid complexion and irritable pulse, I have found the digitalis of great service, several patients recorering entirely under its use; but it is a medicine which requires great care and constant watching.

The sulphate of zinc certainly possesses a decided power over the disease, and I have had three or four patients recover under its use. I seldom give it in larger doses than two grains, and as it is a medicine which may be given without danger, $I$ generally prescribe it in the first instance.

In old and chronic cases, when everything else has failed, I give the oxide of silver, which is one of the most valuable medicines we possess. It requires watching, to prevent its discolouring the skin. But if you do so carefully, and leave it off for about a fortnight at the end of every two months, there is very little danger.

One of the worst cases of epilepsy I ever knew, independent of organic disease, got well under its employment. The patient had been ill fifteen years; he took the medicine for twelve months, and he has been quite well for above six years.

I shall call your attention to this subject again, when I review the progress of our present patient's case, which I hope, for the credit of medical surgery, may prove satisfactory.

\section{SURGICAL CASES.}

By JOHN ERICHSEN, Esq., F.R.C.S., ASIISTANT-SURGEON TO UNIVERSITY COLLEGE HOSPITAL, ETC.

SELECT CASES OF HERNIA.

Strangulated congenital hernia in an adult; with large accumulation of fuid in the tunica vaginalis; operation; cure.

CASE 1.-W. I-, aged twenty, admitted into University College Hospital, Sept. 8, 1819. He states that he has always felt the scrotum somewhat larger on the left than on the right side; a cord appearing to extend from the testis upwards. Nine months ago, in consequence of exertions, he noticed for the first time a distinct tumour in the left groin. This varied somewhat in size, but never disappeared entirely. Did not know that it was a rupture, and had never worn a truss.

Yesterday evening was seized with cramp and pain in the stomach, with vomiting. He applied to a surgeon, who, treat- 